\title{
Influência da Estrutura Química do Co-Monômero nas Propriedades Termomecânicas e Durabilidade de Uniões Adesivas Submetidas à Ação da Água
}

\author{
Filiberto González Garcia, Maria E. Leyva, Alvaro A. A. de Queiroz \\ Instituto de Ciências Exatas, UNIFEI
}

\begin{abstract}
Resumo: A caracterização termomecânica, assim como a durabilidade de juntas adesivas usando diferentes polímeros epoxídicos no estado vítreo foram analisadas. Os polímeros foram baseados no prepolímero do éter diglicidílico do bisfenol A (DGEBA) curado com diferentes aminas alifáticas. Análises termomecânicas no modo de torção foram realizadas para monitorar o fator de perda (tan $\delta$ ), o módulo de armazenamento (G'), e o módulo de perda (G”) em função da temperatura. Os ensaios de adesão foram realizados em uniões de simples sobreposição usando substrato de aço quando submetidas à ação da água a temperatura ambiente, e a $80{ }^{\circ} \mathrm{C}$, com o objetivo de avaliar a durabilidade das juntas adesivas a diferentes temperaturas. As análises termomecânicas evidenciaram diferenças significativas nos valores de $\tan \delta$, G' e G” em função da temperatura para as redes estudadas. As melhores propriedades adesivas são obtidas para uniões polímero-substrato que usam polímeros que apresentem estrutura de rede mais flexível. A durabilidade das juntas adesivas quando submetidas à ação da água causa menor prejuízo à formulação com piperidina. Este comportamento foi associado à baixa tendência de absorver água deste polímero, devido à homopolimerização dos grupos epóxi, que originam uma estrutura de rede com baixa concentração de grupos hidroxila.
\end{abstract}

Palavras-chave: Polímeros epoxídicos, uniões adesivas, caracterização termomecânica, durabilidade.

\section{Influence of Chemical Structure of Co-monomer on Thermomechanical and Durability of Adhesive Joints to the Action of the Water}

\begin{abstract}
The thermomechanical properties, as well as the durability of adhesive joints using different epoxy polymer in the vitreous state was analyzed. The epoxy polymers based on diglycidyl ether of bisphenol A (DGEBA) cured with various aliphatic amines. Rheological tests were used to determine damping ( $\left.\tan \delta=G^{\prime} / G^{\prime}\right)$. The adhesion tests were carried out in steel joints using single lap shear when submit to the action of the water, at ambient temperature and at $80{ }^{\circ} \mathrm{C}$, with the objective of evaluating the durability of the adhesive joints at different temperature. The best adhesive properties were obtained when exists high flexibility of chain between crosslink. The durability of the adhesive joints, when submitted to the action of the water cause smaller damage in the resistance adhesive to the polymer with piperidine. This behavior was associated to the low tendency of absorbing water of this polymer, due to the homopolimerization of the epoxy groups, what origin a network with low concentration of hydroxyl groups.
\end{abstract}

Keywords: Epoxy polymer, adhesive joint, thermomechanical characterizations, durability.

\section{Introdução}

Os polímeros epoxídicos constituem uma das mais importantes classes de polímeros termorrígidos utilizados em adesivos, matrizes para compósitos reforçados, recobrimentos e encapsulados. Para obter alto desempenho usando estes materiais é necessário tanto do ponto de vista do controle do processo de sínteses quanto do processamento, especialmente quando se deseja obter boas propriedades mecânicas e adesivas. Desta maneira é possível o entendimento da relação estrutura-propriedades destes materiais. Numerosos trabalhos têm sido publicados nas últimas décadas em relação a este tópico, especialmente para o caso de redes diepóxi-diaminas ${ }^{[1-8]}$.

A importância do estudo de juntas adesivas, assim como a tecnologia da estrutura das uniões tem sido incrementado, devido a numerosas vantagens do emprego das juntas adesivas em relação à utilização de outros métodos para a união de materiais ${ }^{[9-10]}$. Para obter estruturas resistentes usando juntas adesivas, é importante o estudo do efeito da natureza do adesivo e do substrato, características tais como a rugosidade do substrato e a geometria das uniões ${ }^{[11]}$. Por outro lado, resulta importante o estudo da durabilidade das uniões na presença de água ${ }^{[12-18]}$. Importante, também é obter informação do comportamento das uniões para suportar condições ambientais e sua limitação quando submetidas à ação da água a diferentes temperaturas ${ }^{[19,20]}$.
Neste trabalho, realizamos mudanças na arquitetura molecular dos polímeros epoxídicos, pela utilização de diferentes comonômeros do tipo aminas alifáticas. $\mathrm{O}$ objetivo consistiu em obter informação da relação estrutura-propriedades destas redes poliméricas, assim como estudar a durabilidade de uniões de simples sobreposição produzidas com estes polímeros usando substrato de aço quando submetidas à ação da água a diferentes temperaturas. Visando uma alta funcionalidade no co-monômero foi selecionada a poliamina alifática trietilenotetramina (TETA). Também foram selecionados três co-monômeros do tipo aminas alifáticas com estrutura cíclica tais como piperidina (Pip), 1-(2-aminoetil)piperazina (AEP), isoforono diamina (IPD) as que apresentam diferente funcionalidade e estruturas químicas diferentes.

\section{Experimental}

\section{Materiais}

Os polímeros epoxídicos utilizados estão baseados no prepolímero, produto da reação de epicloridrina e bisfenol-A, (DGEBA) produto técnico DER 331, produzido e comercializado pela Dow Química do Brasil S.A., com quatro co-monômeros do 
tipo aminas alifáticas e cicloalifáticas. Os co-monômeros utilizados foram trietilenotetramina (TETA) produto técnico DEH 24, produzido e comercializado pela da Dow Química do Brasil S.A., piperidina (Pip), 1-(2-aminoetil)piperazina (AEP), isoforono diamina (IPD) produtos (P.A. 99\% de pureza), comercializados pela Sigma-Aldrich do Brasil S.A. Na Tabela 1 são apresentadas as estruturas e algumas propriedades dos monômeros utilizados. A resina foi desidratada cuidadosamente por tratamento a vácuo a $80{ }^{\circ} \mathrm{C}$. Os co-monômeros foram utilizados como recebidos.

\section{Métodos}

\section{Preparação dos polímeros epoxídicos}

Os polímeros foram preparados utilizando proporções estequiométricas (equivalentes de grupos epóxi/equivalentes de hidrogênios amina, e/a = 1) que foi baseado na determinação dos grupos funcionais por métodos de titulação química ${ }^{[22-24]}$. Todas as formulações foram preparadas por adição do co-monômero à resina a temperatura ambiente, com posterior agitação da mistura durante 1 minuto para assegurar a dissolução do co-monômero. Posteriormente as formulações foram vazadas em moldes de silicona na forma de placas retangulares. O programa de cura para os sistemas com, AEP, TETA e IPD, foi realizado em duas etapas. A primeira etapa, 24 horas à temperatura ambiente, e a segunda, em condições isotérmicas que permitem alcançar a conversão máxima, o que foi otimizado por estudos calorimétricos anteriores (DSC) [25]. Para o caso particular do sistema com Pip foi utilizada uma concentração de $5 \mathrm{phr}$ ( $5 \mathrm{~g}$ de piperidina por cada $100 \mathrm{~g}$ de resina), e o programa de cura foi realizado em duas etapas. A primeira etapa, 30 minutos a $60{ }^{\circ} \mathrm{C}$, e a segunda etapa 16 horas a $120^{\circ} \mathrm{C}$. Os materiais retangulares obtidos após o programa de cura foram resfriados lentamente ate à temperatura ambiente. Posteriormente foram usinados até alcançar as dimensões desejadas para a caracterização termomecânica.

\section{Caracterização termomecânica}

A caracterização termomecânica dos polímeros após o programa de cura foi avaliada por reometria no modo de torção usando corpos de prova retangulares $\left(25 \times 10 \times 3 \mathrm{~mm}^{3}\right)$. Para isto foi utilizado um reômetro AR2000 com uma taxa de aquecimento de $2{ }^{\circ} \mathrm{C} / \mathrm{min}$, em um intervalo de 30 a $200{ }^{\circ} \mathrm{C}$, frequiência de $1 \mathrm{~Hz}$. As propriedades no estado vítreo dos materiais foram monitoradas pela medida do fator de perda ( $\left.\tan \delta=G^{\prime} / G^{\prime}\right)$, do módulo de armazenamento $\left(G^{\prime}\right)$, e do módulo de perda (G") em função da temperatura.

O módulo de rigidez elástico borrachoso $\left(\mathrm{G}_{\mathrm{R}}{ }_{\mathrm{R}}\right)$ foi determinado usando a temperatura máxima do pico $(\mathrm{Tm}, \tan \delta)+50{ }^{\circ} \mathrm{C}$ na curva termomecânica de G'vs. T. A massa molecular experimental entre os pontos de entrecruzamento (Mc) foi calculada para os polímeros usando a Equação 1 que descreve a teoria da elasticidade ${ }^{[25]}$. O valor experimental da rede originada com o co-monômero IPD, foi comparado com o valor teórico (Mteor), calculado pelo método divulgado por Bell ${ }^{[26]}$.

$$
\mathrm{Mc}=\rho \mathrm{R} \mathrm{Tm} / \mathrm{G}_{\mathrm{R}}
$$

onde: $\rho$ : densidade do polímero, R: constante dos gases $\left(8,31 \mathrm{~J} \cdot \mathrm{mol}^{-1} \cdot \mathrm{K}^{-1}\right)$

\section{Calorimetria diferencial exploratória (DSC)}

Calorímetro diferencial exploratório (Shimadzu, modelo DSC-60) foi utilizado para determinar a temperatura de transição vítrea $(T g)$ dos polímeros após o programa de cura (amostras com $15 \pm 2 \mathrm{mg}$ ), usando uma taxa de aquecimento de $10{ }^{\circ} \mathrm{C} / \mathrm{min}$ em um intervalo de 30 a $200{ }^{\circ} \mathrm{C} \mathrm{em}$ atmosfera de nitrogênio $\left(20 \mathrm{~cm}^{3} / \mathrm{min}\right)$. O valor de $T g$ foi considerado no ponto médio da mudança da capacidade calórica em relação à linha base da curva calorimétrica.

\section{Ensaios de adesão}

A resistência adesiva foi avaliada em uniões de simples sobreposição as que foram produzidas a partir das formulações epoxídicas usando como substrato a liga de aço A36. Para este propósito o ensaio foi realizado segundo o protocolo da norma ASTM D 1002-01.

Para melhorar o desempenho das juntas adesivas o substrato metálico foi tratado. $\mathrm{O}$ tratamento consistiu em:

1) Limpeza com solvente: o substrato metálico foi tratado com 1,1,1-tricloroetileno.

2) Tratamento mecânico: a superfície das peças metálicas a serem coladas foram tratadas com granalhas de aço G40 utilizando uma máquina de jateamento por sucção usando uma pressão no jato de $6,5 \mathrm{~kg} \cdot \mathrm{cm}^{-1}$. O tamanho das granalhas de aço utilizadas foi na faixa de 0,30 a $0,42 \mathrm{~mm}$.

Tabela 1. Estrutura química e características dos monômeros.

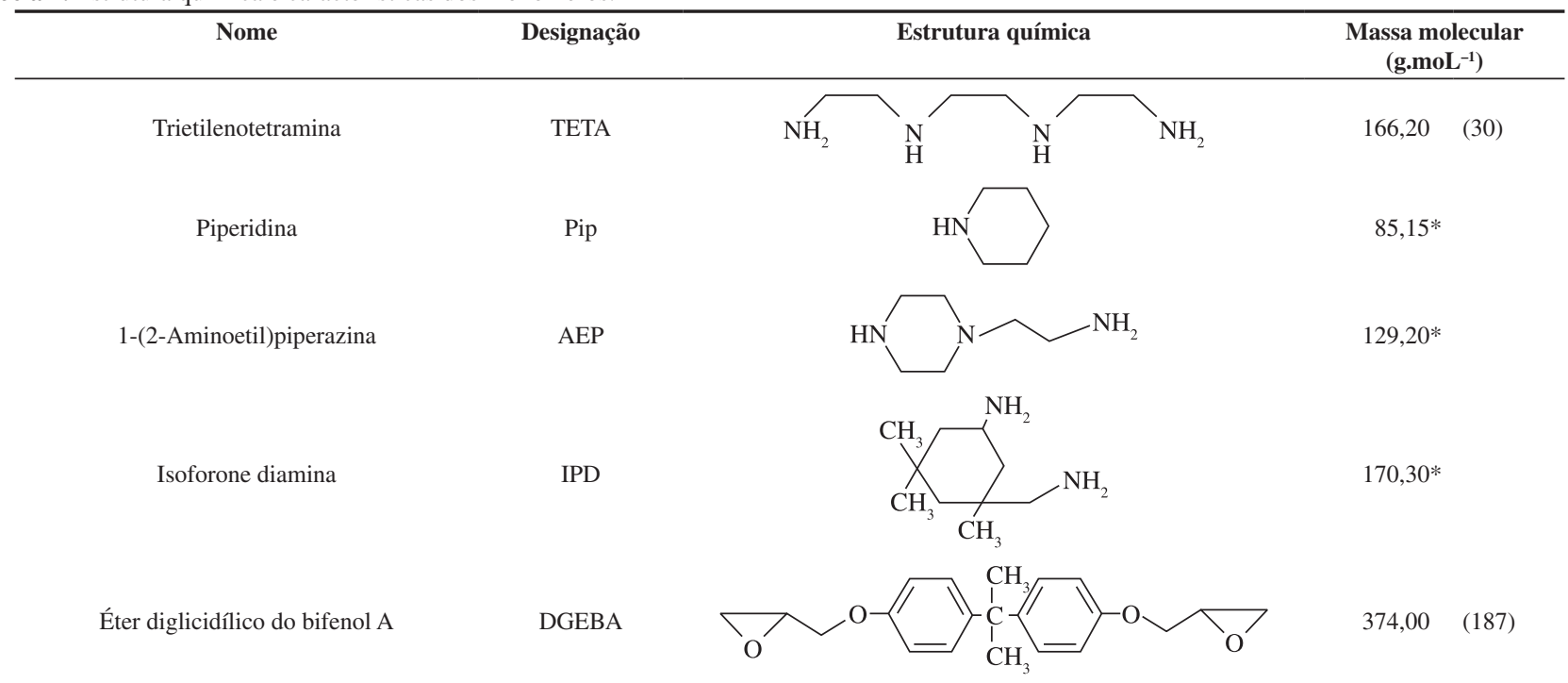

\footnotetext{
*Valores teóricos, os valores entre parênteses correspondem ao equivalente de grupos epóxi e equivalentes de hidrogênio do tipo amina. No caso do co-monômero TETA foi encontrado em trabalho anterior que sua funcionalidade corresponde 5,54 ${ }^{[22]}$.
} 
3) Secado e conservação das peças metálicas: após o tratamento mecânico as peças foram secas cuidadosamente com um fluxo de ar seco. Logo foram conservadas em câmara seca, até a preparação das juntas adesivas.

Para a aplicação dos adesivos foi desenhado um molde específico para este propósito. Depois do tratamento as peças de aço foram montadas como uniões de simples sobreposição $(102 \times 25 \times 1,6 \mathrm{~mm})$ usando uma extensão de colagem de $312,5 \mathrm{~mm}^{2}$. A pressão de contato aplicada nas uniões foi sempre a mesma, o que assegurou obter uma espessura da camada adesiva uniforme $(0,16 \pm 0,05 \mathrm{~mm})$. Os ensaios foram realizados depois de submeter ás uniões ao programa de cura. Para os sistemas com, AEP, TETA e IPD, foi realizado em duas etapas. A primeira etapa para os três sistemas, 24 horas à temperatura ambiente, e a segunda, 2 horas a $130{ }^{\circ} \mathrm{C}$ para os dois primeiros sistemas, e para o último 2 horas a $160{ }^{\circ} \mathrm{C}$. Para o caso particular do sistema com Pip foi utilizada uma concentração de $5 \mathrm{phr}$ ( $5 \mathrm{~g}$ de piperidina por cada $1 \mathrm{~g}$ de resina), e o programa de cura foi realizado em duas etapas. A primeira etapa, 30 minutos a $60{ }^{\circ} \mathrm{C}$, e a segunda etapa 16 horas a $120{ }^{\circ} \mathrm{C}$. Para este ensaio foi utilizada uma máquina universal de ensaios (Shimadzu AG I $100 \mathrm{kN}$ ) usando uma taxa do travessão de $1,27 \mathrm{~mm} / \mathrm{min}$. A resistência adesiva é expressa em $\mathrm{MPa}$, e calculado pela divisão do valor da carga na ruptura pela área de colagem. Os ensaios foram conduzidos a $22{ }^{\circ} \mathrm{C}$, e os valores reportados foram a media de 10 determinações.

\section{Durabilidade das uniões submetidas à ação da água}

As uniões de simples sobreposição produzidas com os polímeros e o substrato metálico após o programa de cura foram submersas em água destilada a temperatura ambiente $\left(24 \pm 4{ }^{\circ} \mathrm{C}\right)$ e a $80{ }^{\circ} \mathrm{C}$ por diferentes tempos. $\mathrm{O}$ ensaio consistiu em submergir as juntas adesivas em água destilada a temperatura ambiente, e ou a $80{ }^{\circ} \mathrm{C}$, e posteriormente retirar-las a diferentes tempos para cada temperatura. Antes do ensaio as uniões foram mecanicamente secas com pano de algodão e ar seco.

\section{Resultados e Discussão}

\section{Propriedades termomecânicas}

Nas Figuras 1, 2 e 3 são apresentadas os comportamentos termomecânicos no modo de torção, o fator de perda $\left(\tan \delta=G^{\prime \prime} / G^{\prime}\right)$, o módulo de armazenamento (G'), e o módulo de perda (G”) em função da temperatura, respectivamente para as redes estudadas. $\mathrm{Na}$ Tabela 2 estão sumarizadas as propriedades termomecânicas dos polímeros.

Nas Figuras 1, 2 e 3 pode ser observado que existe diferença significativa nos valores de $\tan \delta$, G' e G' em função da temperatura para as redes estudadas. Este comportamento está relacionado, às diferenças entre os valores de $T g$ dos polímeros. Em particular, para o polímero DGEBA/Pip, os valores de $\tan \delta$, G' e G" resultam sempre inferiores, quando comparadas às dos outros polímeros. Isto está relacionado ao menor valor de $T g$ desta rede, quando comparado ao valor das outras (Tabela 2). Este comportamento, também pode ser explicado como resultado da menor rigidez desta rede, devido à formação de uma estrutura tridimensional mais flexível (Figura 4).

Este comportamento também é resultado das diferenças entre os mecanismos de polimerização que originam estas redes. Para os sistemas DGEBA/AEP, DGEBA/TETA e DGEBA/IPD o mecanismo de polimerização que ocorre, corresponde à clássica polimerização por etapas, onde é utilizada relação estequiométrica entre os grupos funcionais, e é utilizado um programa de cura adequado que garante a conversão máxima nestes sistemas. Nestes sistemas, os diferentes valores de $T g$, são o resultado da diferente

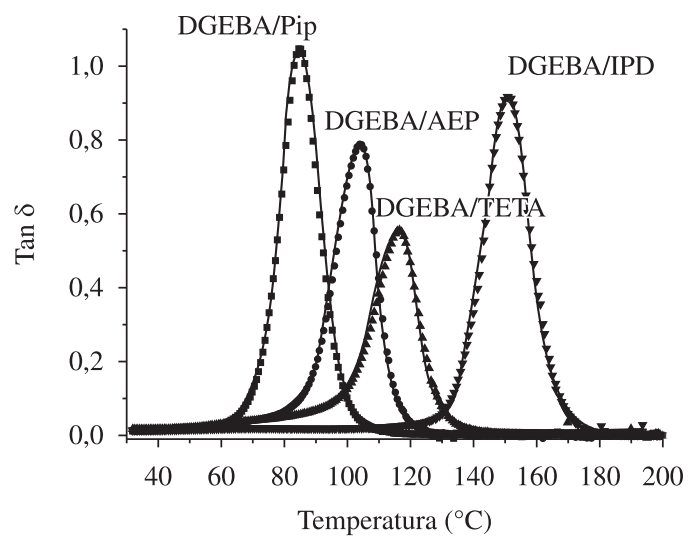

Figura 1. Análises termomecânicas no modo de torção. Fator de perda ( $\tan \delta$ ) em função da temperatura.

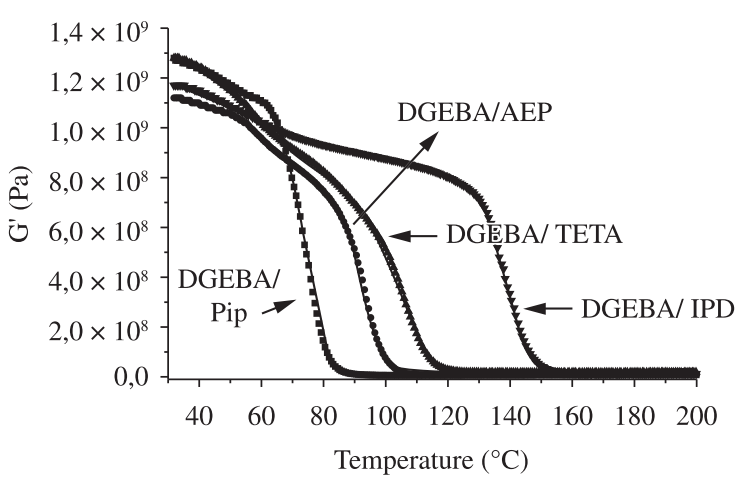

Figura 2. Curva termomecânica de módulo de estocagem $\left(\mathrm{G}^{\prime}\right)$ em função da temperatura $(\mathrm{T})$.

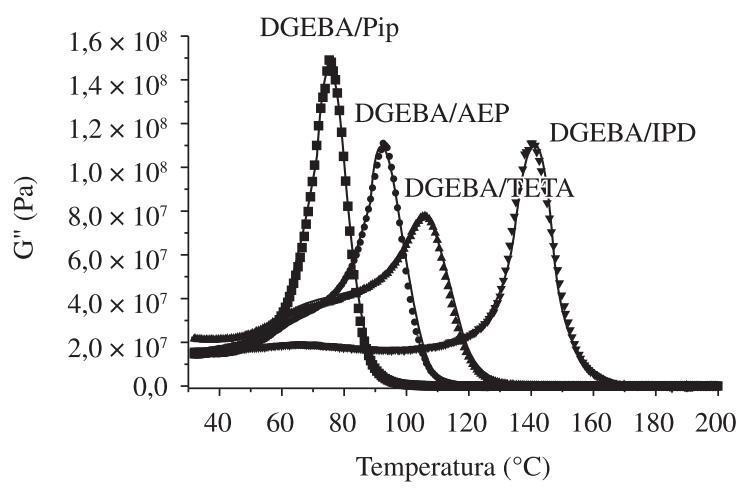

Figura 3. Curva termomecânica do módulo de perda (G”) em função da temperatura $(\mathrm{T})$.

estrutura química do co-monômero, o que provoca mudanças nas estruturas das redes.

Para o caso particular, do polímero originado com o co-monômero piperidina, não ocorre somente o mecanismo de polimerização por etapas, como nos três sistemas anteriores. Neste caso, a polimerização ocorre por dois mecanismos diferentes. Primeiro ocorre o mecanismo de polimerização por etapas, até o consumo do hidrogênio secundário da amina, e depois em excesso de grupos epóxi, ocorre o mecanismo de homopolimerização dos grupos epoxídicos via mecanismo aniônico ${ }^{[27-29]}$. Isto origina estruturas de cadeias do tipo poliéter relativamente longas e flexíveis, e como conseqüência um menor valor de $T g$, e maior valor de Mc (maior separação entre os pontos de entrecruzamento, 
Tabela 2. Propriedades reológicas dos polímeros usando o modo de torção.

\begin{tabular}{ccccc}
\hline Polímeros & $\begin{array}{c}\mathbf{T m}(\tan \delta) \\
\left({ }^{\circ} \mathbf{C}\right)\end{array}$ & $\begin{array}{c}\mathbf{G}_{\mathbf{R}}^{\prime} \\
(\mathbf{M P a})\end{array}$ & $\begin{array}{c}\mathbf{M c} \\
(\text { experimental })\end{array}$ & $\begin{array}{c}\mathbf{M}_{\text {teor }} \\
\text { (teórico) }\end{array}$ \\
\hline DGEBA/Pip & 85,1 & 6,4 & 563 & - \\
DGEBA/AEP & 104,1 & 8,6 & 412 & - \\
DGEBA/TETA & 116,3 & 17,0 & 213 & - \\
DGEBA/IPD & 150,9 & 14,4 & 271 & 277 \\
\hline
\end{tabular}

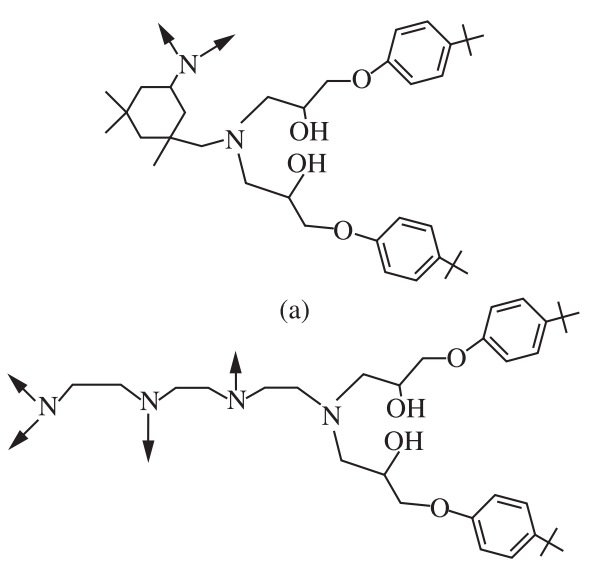

(b)
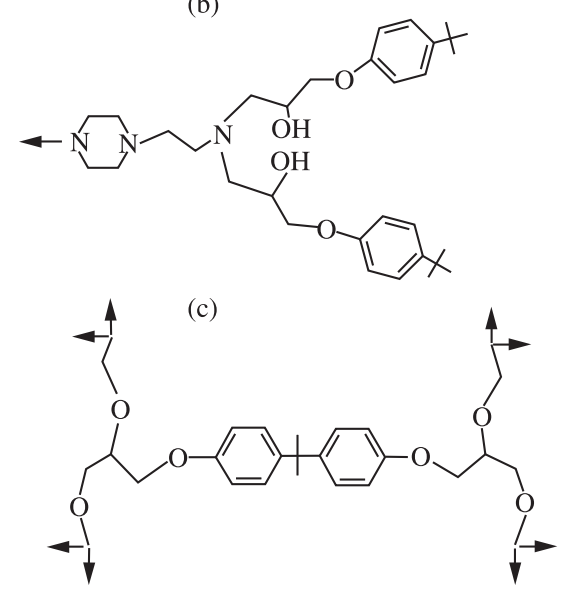

(d)

Figura 4. Estrutura química das redes. a) com isoforono diamina (IPD), b) com trietilenotetramina (TETA), c) com 1-(2-aminoetil)piperazina (AEP), e d) com piperidina.

ver Tabela 2) e, portanto esta rede apresenta a menor densidade de entrecruzamento, quando comparadas às outras.

Na Tabela 2 são apresentados os valores experimentais da massa molecular entre os pontos de entrecruzamento (Mc), determinados a partir dos dados termomecânicos. Estes resultados parecem coerentes para as redes estudadas. Exceto para o caso do polímero originado com TETA, onde um valor maior de Mc era esperado, quando comparado ao valor obtido para a rede com IPD. Esta afirmação está baseada à presença na molécula de TETA de uma seqüência de estruturas etilênicas com maior mobilidade, quando comparado à estrutura cíclica, mais rígida na molécula de IPD. Este comportamento pode ser resultado, de que este comonômero (TETA), foi demonstrado em trabalhos anteriores que está constituído por uma mistura de poliaminas ${ }^{[21,30]}$, e por outro lado, é possível que o método experimental não possa detectar pequenas mudanças nas medidas do módulo $\left(\mathrm{G}_{\mathrm{R}}^{\prime}\right)$ a altas temperaturas. Entretanto, o valor experimental da massa molecular entre os pontos
Tabela 3. Propriedades térmicas e resistência adesiva das uniões nos ensaios de adesão usando uniões de simples sobreposição.

\begin{tabular}{ccc}
\hline Polímeros & $\begin{array}{c}\boldsymbol{T g} * \\
\left({ }^{\circ} \mathbf{C}\right)\end{array}$ & $\begin{array}{c}\text { Resistência adesiva } \\
(\mathbf{M P a})\end{array}$ \\
\hline DGEBA/Pip** & 80 & $21,0 \pm 0,4$ \\
DGEBA/AEP*** & 115 & $19,9 \pm 0,8$ \\
DGEBA/TETA*** & 124 & $16,6 \pm 0,8$ \\
DGEBA/IPD*** & 155 & $17,5 \pm 0,5$ \\
\hline *Determinada pelas análises calorimétricas no modo dinâmico (DSC). \\
**Para o sistema foi utilizada uma concentração de $5 \mathrm{phr}(5 \mathrm{~g}$ de \\
piperidina por cada 100 g de resina), e o programa de cura foi realizado \\
em duas etapas. A primeira, 30 minutos a $60^{\circ} \mathrm{C}$, e a segunda 16 horas \\
a $120{ }^{\circ} \mathrm{C}$. ***Para os sistemas foi utilizada relação estequiométrica, \\
e o programa de cura foi realizado em duas etapas. A primeira etapa, \\
24 horas à temperatura ambiente, e a segunda, 2 horas a $130{ }^{\circ} \mathrm{C}$.
\end{tabular}

de entrecruzamento, para a rede originada pelo co-monômero IPD está em perfeito acordo, com o valor teórico. Esta concordância entre os valores experimentais, e teóricos para o sistema DGEBA/ IPD justifica que os valores de massas moleculares entre os pontos de entrecruzamento experimentais (Mc), possam ser considerados válidos para efeitos de comparação para as redes estudadas.

\section{Propriedades adesivas}

A Tabela 3 mostra a resistência adesiva das uniões de simples sobreposição produzidas com os diferentes polímeros usando o substrato metálico. Os valores de resistência adesiva estão relacionados com os valores de $T g$ dos diferentes polímeros. Este resultado é similar ao comportamento termomecânico, previamente discutido. O sistema DGEBA/IPD exibe alto valor de $T g$, e menor resistência adesiva nas uniões de simples sobreposição. Entretanto, os sistemas DGEBA/Pip e DGEBA/AEP mostraram os melhores desempenhos na resistência adesiva, devido aos menores valores de $T g$ como resultado de uma estrutura de rede mais flexível. O maior desempenho adesivo destas duas redes está relacionado ao baixo valor de $T g$, como consequiência de uma estrutura de rede, com maior massa molecular entre os pontos de entrecruzamento (Mc). Isto origina uniões adesivas com maior flexibilidade, o que garante uniões mais resistentes para suportar as tensões axiais.

Na Figura 4 são apresentadas as estruturas químicas das redes originadas para os diferentes co-monômeros. Os sistemas DGEBA/ Pip e DGEBA/AEP apresentam maior flexibilidade, e baixo valor de $T g$, quando comparados aos sistemas DGEBA/ TETA e DGEBA/IPD. Os polímeros originados com os comonômeros TETA e IPD mostram alta densidade de entrecruzamento (menor valor de Mc). Entretanto, o polímero com TETA exibe uma estrutura mais flexível, quando comparada à estrutura rígida, do polímero originado com IPD (Figura 4). Estes resultados permitem afirmar que as melhores propriedades adesivas são obtidas para uniões polímero-substrato produzidas com polímeros que apresentem estrutura de rede mais flexível, o que pode ser obtido selecionando redes com baixo valor de $T g$.

O comportamento entre as propriedades termomecânicas e adesivas estão relacionados à estrutura de rede. Neste caso, as redes originadas com os co-monômeros AEP, TETA e IPD são chamadas de "redes fechadas", as que são o resultado do mesmo mecanismo de polimerização por etapas. Além disso, são formadas por misturas estequiométricas de monômeros os que conseguem reagir até conversão máxima $\left(T g_{\max }\right)$. Para o caso particular da rede originada com o co-monômero piperidina ocorrem dois mecanismos diferentes de polimerização, como foi mencionado anteriormente. $\mathrm{O}$ que origina uma estrutura de rede com cadeias mais longas, e por isto apresenta um menor valor de $T g$, como consequência de uma 
maior flexibilidade das cadeias, e maior valor de Mc e, portanto a menor densidade de entrecruzamento. Neste caso, parece possível aceitar, baseado nos resultados obtidos neste trabalho, que as propriedades termomecânicas, e adesivas estão relacionadas com a estrutura molecular das redes estudadas. Entretanto, é importante ressaltar que a estrutura da rede do sistema DGEBA/Pip é bem diferente quando comparada às outras.

\section{Durabilidade das uniões submetidas à ação da água}

A Figura 5 mostra a resistência adesiva das uniões de simples sobreposição submetidas à ação da água a temperatura ambiente. Um pequeno decréscimo pode ser observado na resistência adesiva quando aumenta o tempo de imersão em água até alcançar um valor estável em um tempo de 45 dias para os sistemas DGEBA/TETA, DGEBA/IPD e DGEBA/AEP que representa uma diminuição da resistência adesiva de 8,3, 7,5 e 8,0\%, respectivamente. Entretanto para o sistema DGEBA/Pip se observa que alcança um valor estável em um tempo de 15 dias após a imersão em água que representa uma diminuição da resistência adesiva de 1,4\%. A literatura reporta, que a atividade da água após a imersão de uma amostra de polímero, pode ser analisada por curvas termogravimétricas ${ }^{[6]}$. Onde o comportamento de um polímero imerso em água, pode apresentar um aumento gradativo de massa até alcançar um valor máximo. Para as juntas adesivas imersas na água este comportamento pode

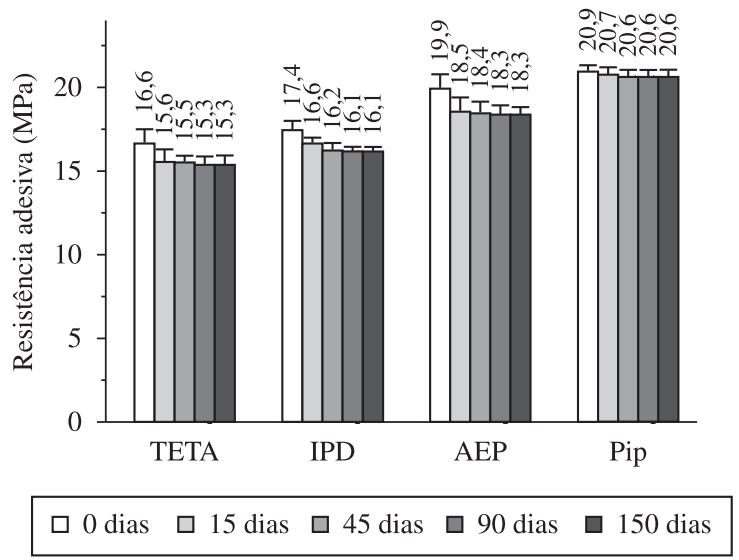

Figura 5. Resistência adesiva das uniões de simples sobreposição produzidas com os polímeros e o substrato de aço depois de submetidas à ação da água a temperatura ambiente.

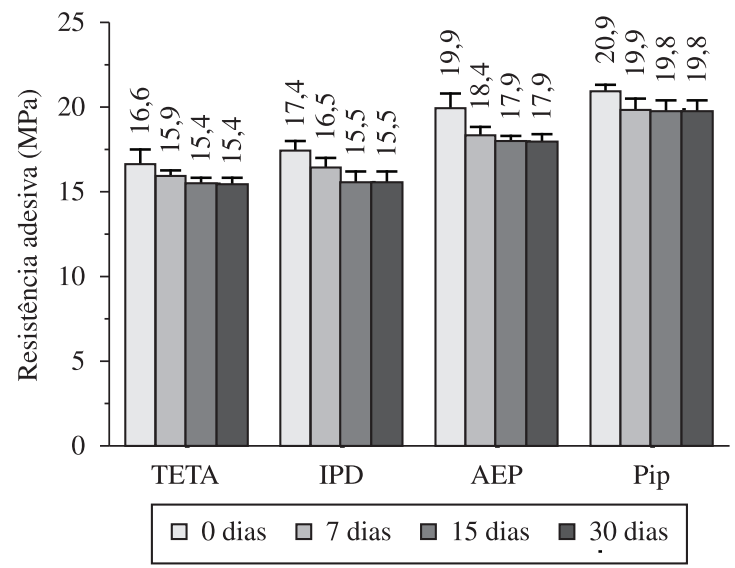

Figura 6. Resistência adesiva das uniões de simples sobreposição produzidas com os polímeros e o substrato de aço depois de submetidas à ação da água a $80{ }^{\circ} \mathrm{C}$. estar relacionado à diminuição da resistência adesiva até alcançar um valor estável onde ocorre a saturação da água no polímero. Segundo a literatura isto indica a existência de processos químicos irreversíveis, hidrólises, o que provoca mudanças físicas, prejuízo nas propriedades, e a existência de micro-cavidades devido à capacidade do material de adsorver água.

Nas juntas adesivas submetidas à ação da água a $80{ }^{\circ} \mathrm{C}$, foi observado que a resistência adesiva também diminui com o tempo de imersão em água, e depois alcança um valor estável em um tempo de 15 dias (Figura 6) para os sistemas DGEBA/DETA, DGEBA/ IPD e DGEBA/AEP que representa uma diminuição da resistência adesiva de 8,7, 10,9 e 10,0\%, respectivamente. Entretanto, para o sistema DGEBA/Pip o valor estável é alcançado em um tempo de 7 dias que representa uma diminuição da resistência adesiva de 5,2\%. Este comportamento era esperado devido a que a possível saturação do polímero pela água deve acontecer em um tempo menor com o aumento da temperatura. Por outro lado, este comportamento da resistência adesiva das uniões submetidas à ação da água a $80^{\circ} \mathrm{C}$ confirma que este tipo de adsorção de água ocorre nos polímeros estudados.

Importante ressaltar que em todas as uniões submetidas à ação da água ocorre uma diminuição na resistência adesiva com o tempo de exposição. A magnitude do prejuízo é diferente para as uniões preparadas para o sistema DGEBA/Pip. O decréscimo da resistência adesiva para as uniões produzidas com o co-monômero piperidina resultou a mais baixa. Isto é conseqüência de uma estrutura de rede formada pela homopolimerização dos grupos epóxi, o que origina uma estrutura com baixa concentração de grupos hidroxila, e por isto apresenta uma baixa tendência para absorver água ${ }^{[11]}$.

\section{Conclusões}

As propriedades termomecânicas e adesivas das uniões de simples sobreposição quando submetidas à ação da água dos polímeros epoxídicos mostraram-se dependentes da estrutura molecular das redes. O sistema DGEBA/IPD exibe alto valor de $T g$, e menor resistência adesiva nas uniões de simples sobreposição. Entretanto, os sistemas DGEBA/Pip e DGEBA/AEP mostraram os melhores desempenhos na resistência adesiva, devido aos menores valores de $T g$ como resultado de uma estrutura de rede mais flexível. Além disso, as uniões produzidas com sistema DGEBA/Pip mostraram maior durabilidade quando submetidas à ação da água como conseqüência de uma baixa concentração de grupos hidroxila.

\section{Agradecimentos}

Os autores agradecem o apoio financeiro das entidades brasileiras CNPq e FAPEMIG ref. TEC 00242/07. Agradecemos ao Dr. Terri Chen da TA Instruments-Waters LLC e à equipe da dp UNION Instrumentação Analítica e Científica pela gentileza para a realização das análises termomecânicas em seus laboratórios.

\section{Referências Bibliográficas}

1. Gupta,V. B; Dizal, L. T. \& Lee, C. Y. C. - Polym. Eng. Sci., 25, p.812 (1985). http://dx.doi.org/10.1002/pen.760251305

2. Jordan, C.; Galy; J. \& Pascault, J.P.- J. Appl. Polym. Sci., 46, p.859 (1992). http://dx.doi.org/10.1002/app.1992.070460513

3. Amdouni, N.; Sautereau, H; Gerard, J. F. \& Pascault, J. P.- Polymer, 31, p.1245 (1990). http://dx.doi.org/10.1016/0032-3861(90)90215-K

4. Misra, S. C.; Manson, J. A. \& Sperling, L. H. - ACS Adv. Chem. Ser., 114, p.137 (1979).

5. Vaskil, U. \& Martin, G. C. - J. Appl. Polym. Sci., 46, p.2089 (1992). http://dx.doi.org/10.1002/app.1992.070461204 
6. Pascault, J. P.; Sautereau, H.; Verdu, J. \& Williams, R. J. J. "Thermosetting polymers", Marcel Dekker, New York (2002). http:// dx.doi.org/10.1201/9780203908402

7. Urbancewski, E.; Galy, J.; Gerard, J. F.; Pascault, J. P.\& Sautereau, H. - Polym. Eng. Sci., 31, p.1572 (1991).

8. Grillet, A. C.; Galy, J.; Gerard, F. \& Pascault, J. P.- Polymer, 32, p.1885 (1991). http://dx.doi.org/10.1016/0032-3861(91)90380-2

9. Higgins, A. - Int. J. Adhesion \& Adhesives, 20, p.367 (2000). http:// dx.doi.org/10.1016/S0143-7496(00)00006-3

10. Pearce, P.; Ennis, B. C.; Grabovac, I. \& Morris, C. - J. Adhesion., 47, p.123 (1994). http://dx.doi.org/10.1080/00218469408027094

11. Prolongo, S. G.; Del Rosario, G. \& Ureña, A. - Int. J. Adhesion \& Adhesives, 26, p.125 (2006). http://dx.doi.org/10.1016/j. ijadhadh.2005.02.004

12. Bhowmik, S.; Bonin, H. W, Bui, V. T. \& Weir, R. D. - Int. J. Adhesion \& Adhesives, 26, p.400 (2006). http://dx.doi.org/10.1016/j. ijadhadh.2005.05.004

13. Digby, R. P.\& Packham, D. E. - Int. J. Adhesion \& Adhesives, 15, p.61 (1995). http://dx.doi.org/10.1016/0143-7496(95)98739-9

14. Critchlow, G. W. \& Brewis, D. M. - Int. J. Adhesion \& Adhesives, 15, p.173 (1995). http://dx.doi.org/10.1016/0143-7496(95)91628-J

15. Popineau, S.; Rondeau-Mouro, C.; Sulpice-Gaillet, C. \& Shanahan, M. E. R. - Polymer, 46, p.10733 (2005). http://dx.doi.org/10.1016/j. polymer.2005.09.008

16. Papanicolaou, G. C.; Kosmidou, T. V.; Vatalis, A. S. \& Delides, C. G. - J. Appl. Polym. Sci., 99, p.1328 (2006). http://dx.doi.org/10.1002/ app. 22095

17. Abdelkader, A. F. \& White, J. R. - J. Appl. Polym. Sci., 98, p.2544 (2005). http://dx.doi.org/10.1002/app.22400

18. Maggana, C. \& Pissi, P.- J. Appl. Polym. Sci., 37, p.1165 (1999). http:// dx.doi.org/10.1002/(SICI)1099-0488(19990601)37:11\%3C1165::AIDPOLB11\%3E3.0.CO;2-E
19. Armstrong, K. B. - Int. J. Adhesion \& Adhesives, 17, p.89 (1997). http://dx.doi.org/10.1016/S0143-7496(96)00038-3

20. Bowditch, M. R. - Int. J. Adhesion \& Adhesives, 16, p.73 (1996). http:// dx.doi.org/10.1016/0143-7496(96)00001-2

21. González Garcia, F.; Miguez, E. \& Soares, B. G. - Polímeros, 15, p.261 (2005).

22. González Garcia, F.; Silva, P.M.; Soares, B. G. \& Rieumont, J. - Polym. Test, 26, p.95 (2007).

23. American Society for Testing and Materials - ASTM. - "ASTM D1652: Método para determinação do conteúdo de epóxi em resinas epoxídicas", ASTM (1997).

24. González Garcia, F.; Soares, B. G.; Pita, V. J. R. R; Sánchez, R. \& Rieumont, J. - J. Appl. Polym. Sci., 106, p.2047 (2007).

25. De'Neve, B. \& Sananhan, M. E. R. - J. Adhes., 49, p.165 (1996). http:// dx.doi.org/10.1080/00218469508014353

26. Bell, J. P. - J. Polym. Sci., 6, p.417 (1970).

27. Cuadrado, T. R.; Almaraz, A. \& Williams, R. J. J. - "Curing of Epoxy Resins with Piperidine”, in: Crosslinked Epoxies, B. Sedláček J. Kahovec (ed.), De Gruyter, Berlin, p.170 (1987).

28. Galego, N.; Vázquez, A. \& Williams, R. J. J. - Polymer, 35, p.857 (1994). http://dx.doi.org/10.1016/0032-3861(94)90886-9

29. González Garcia, F.; Ortiz, P.\& Galego, N. - Polym. Int., 42, p.163 (1997).

30. González Garcia, F.; Miguez, E. \& Tavares, M. I. B. - Polímeros, 18, p.45 (2008)

Enviado: $26 / 08 / 10$

Reenviado: $26 / 10 / 10$

Aceito: $07 / 12 / 10$

DOI: $10.1590 / \mathrm{S} 0104-14282011005000057$ 